\title{
INFLUENCIA DEL CONTACTO PRECOZ, NACIONALIDAD, TIPO DE PARTO Y PREMATURIDAD EN LA LACTANCIA MATERNA
}

\section{INFLUENCE OF EARLY SKIN-TO-SKIN CONTACT, NATIONALITY, TYPE OF BIRTH AND PREMATURE BIRTH IN BREAST FEEDING}

\author{
TítUlO CORTO: INFLUENCIA DEL CONTACTO PRECOZ, NACIONALIDAD, TIPO DE PARTO Y \\ PREMATURIDAD
}

Ana Belén Laviña Castan ${ }^{1}$

Recibido en Junio 11 de 2014

Aceptado en Agosto 15 de 2014

\section{Resumen}

Conocer si en el HUMS existe asociación entre lactancia materna y nacionalidad, prematuridad, tipo de parto, contacto precoz piel con piel. Estudio de cohortes, en una muestra de 541 puérperas del HUMS en octubre-noviembre 2011. Las variables: nacionalidad, tipo de parto, contacto precoz, parto prematuro e inicio lactancia materna se recogieron antes del alta hospitalaria mediante cuestionario. A los dos meses postparto se contactó telefónicamente con las madres para conocer el tipo de lactancia en ese momento. El 70,32 \% iniciaron la lactancia materna en las dos primeras horas postparto y el 55,58\% daban Lactancia materna exclusiva (LME) a los dos meses. Influyen en la lactancia materna el tipo de parto, contacto precoz y parto prematuro. La probabilidad de lactancia materna es 3,65 veces más alta si se ha realizado contacto precoz (RR 3,65; IC 95\% 2,72-4,88; $p=0,001$ ). El inicio de lactancia materna en las 2 primeras horas y la nacionalidad española influye positivamente en la LME a los 2 meses. Las prácticas hospitalarias en el postparto deben promover el contacto precoz madre-recién nacido y el inicio de lactancia materna en las dos primeras horas, principalmente en las cesáreas.

Palabras clave: Lactancia materna, nacionalidad, cesárea, parto prematuro.

\section{Abstract}

To know if there is any relationship between breast feeding and nationality, premature birth, type of birth and early skin-to-skin contact at Hospital Universitario Miguel Servet. Cohort study in a simple of 541 puerperal women at Hospital Miguel Servet, Zaragoza, from October to November 2011.The variables nationality, type of birth, early skin-to-skin contact, premature birth and beginning of breast feeding were collected before hospital discharge by means of a questionnaire.Two months after the birth, mothers were phoned to know the type of breastfeeding until

1. Enfermera Especialista en Obstetricia y Ginecología (Matrona). Hospital Universitario Miguel Servet de Zaragoza (HUMS). Zaragoza, España. Correo: anabelenlavina@gmail.com 
that moment. 70,32\% of women began breastfeeding within the first two hours after the birth and 55,58\% were breastfeeding exclusively after two months. Type of birth, early skin-to-skin contact and premature birth have an influence on breastfeeding. The probability of breastfeeding is 3,65 times higher if there has been early skin-to-skin contact (RR 3,65; IC 95\% 2,72-4,88; p=0,001). Beginning of breastfeeding within the first two hours and the Spanish nationality have a positive influence in breastfeeding in two months. Postpartum practices at hospitals must promote the early contact between the newborn and the mother, and the beginning of breastfeeding within the first two hours, especially in cesarean sections.

Keywords: Breastfeeding, nationality, cesarean section, premature birth.

\section{Introducción}

A pesar de los beneficios reconocidos a la lactancia materna (LM) ${ }^{1}$ en España estamos muy por debajo de las recomendaciones de la $\mathrm{OMS}^{2}$ según los últimos datos de ámbito nacional ${ }^{3}$.

Actualmente existe un abandono prematuro de Lactancia materna ${ }^{4}$. Ciertos estudios encuentran el mayor abandono entre los 15 días y los 2 meses $^{5}$, siendo al segundo mes donde se produce el mayor cese de lactancia ${ }^{6}$

El análisis de las causas del abandono prematuro de la lactancia debería incluir las intervenciones de los servicios de salud y las características sociales, culturales y económicas?

Respecto a las intervenciones de los servicios de salud se han ido adoptando iniciativas para promocionar la LM, entre las que destaca el documento elaborado por la OMS y UNICEF en 1989 “ los 10 pasos para una feliz lactancia natural" siendo la base de la Iniciativa Hospital Amigo de los Niños (IHAN) ${ }^{8}$. Actualmente denominada "Iniciativa para la Humanización de la Asistencia al Nacimiento y la Lactancia" interpretándose el paso cuarto como: Colocar a los bebés en contacto piel con piel con sus madres inmediatamente después del parto, por lo menos durante una hora.

116

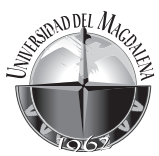

El momento ideal para el inicio de la LM es el postparto inmediato, a ser posible durante las 2 primeras horas de vida, momento en que el bebé se encuentra más reactivo ${ }^{1}$.En el inicio e instauración de LM son fundamentales las rutinas asistenciales adecuadas, como su inicio precoz y el contacto continuado ${ }^{10}$; pero las rutinas hospitalarias van perdiendo relevancia a medida que trascurren los meses ${ }^{11}$, por este motivo se desea estudiar la LM antes de que interfieran otros factores como son la reincorporación al trabajo y la introducción de alimentos al bebé que producen abandono de lactancia materna entre los 3 y 6 meses $^{1}$, señalar que las diferencias en la exclusividad y duración de la lactancia materna dependen en gran medida de las creencias culturales ${ }^{12}$.

España es el segundo país de la Unión Europea con más población extranjera ${ }^{13}$ En 2011 el 12,2 \% de la población censada eran extranjeros, siendo el $48,3 \%$ mujeres ${ }^{14}$; en Aragón la población extranjera es el 12,7\% aunque hay una ligera tendencia al descenso en 2011 las cifras se mantienen respecto a los tres años anteriores ${ }^{15}$.

Un 19,1\% de los partos de 2011 en España fueron de madre extranjera ${ }^{13}$. El porcentaje de LM, tanto natural como mixta, es superior en madres extranjeras, según los últimos datos disponibles de $2006^{3}$.

Este dato junto con la influencia de la "cultura de la lactancia materna" según la población de origen ${ }^{16} y$ la relevancia de las prácticas hospitalarias ${ }^{11}$ justifican este estudio que tiene como propósito analizar el inicio y mantenimiento de la lactancia materna exclusiva (LME) a los dos meses postparto en función de la nacionalidad y el contacto precoz con el recién nacido.

El objetivo principal de este estudio es conocer si en el HUMS existe asociación entre la lactancia materna (tanto su inicio en las dos primeras horas tras el parto como su continuación de forma exclusiva a los dos meses) y nacionalidad, prematuridad, tipo de parto, contacto precoz piel con piel en la primera hora tras el parto. Por otra parte, analiza la relación entre inicio de lactancia materna en las dos primeras horas tras el 
parto y la continuación con lactancia materna exclusiva a los dos meses.

\section{Materiales y Métodos}

Se realizó un estudio de cohortes (Longitudinal, prospectivo) en el Hospital Universitario Miguel Servet de Zaragoza (HUMS). Hospital terciario cuya maternidad asiste a las Áreas de salud 2 y 5 del mapa sanitario de la Comunidad Autónoma de Aragón. Es centro de referencia de Neonatología y Cirugía Pediátrica de la comunidad Autónoma de Aragón, La Rioja y Soria. En este hospital se atendieron un total de 4521 partos en el año 2011; se estudiaron todas las madres que dieron a luz en los meses de octubre y noviembre de 2011 en el HUMS de Zaragoza y se efectuó un muestreo consecutivo estando la muestra compuesta por todas las madres que cumplían los criterios de inclusión.

\section{Criterios de inclusión y exclusión}

Fue criterio de inclusión haber tenido en el HUMS un hijo vivo con una edad gestacional igual o superior a 22 semanas.

Se consideraron criterios de exclusión:

- Mujeres con patología psiquiátrica previa (confirmada en la historia clínica).

- En el parto actual: muerte neonatal

- Dificultad para comprender el castellano.

- No deseen participar en el estudio y/o no firmen consentimiento del estudio.

\section{Variables y recogida de datos}

- Nacionalidad: española, extranjera. (En extranjeras indicar: latinoamericana, Europa Este, magrebí o subsahariana, asiáticas, Unión europea).

- Prematuridad: sí/no. (El parto pretérmino ocurre antes de las 37 semanas de gestación ${ }^{17}$.

- Tipo de parto: Eutócico/distócico (instrumental y cesárea).

- Contacto piel con piel en la primera hora de vida del bebé9.

- Inicio temprano de lactancia materna en las dos primeras horas tras el parto ${ }^{1}$ (pudiendo ser toma directamente del pecho o administración del calostro extraído con sacaleches).

- Tipo lactancia a los 2 meses postparto: LME /LM mixta.
Esta última variable no figura en el cuestionario, se recogió contactando telefónicamente con la madre a los 2 meses postparto. En este estudio se habla de LME a los 2 meses postparto si el bebé toma exclusivamente lactancia materna, no recibiendo nada de leche de fórmula. La recogida de datos se realizó tras la firma del consentimiento informado por la madre, mediante un cuestionario elaborado según la bibliografía consultada.

Se realizó una prueba piloto en un $5 \%$ de las madres que dieron a luz en el HUMS de Zaragoza en septiembre de 2011 (2 meses antes de la realización del estudio) con el fin de validar el cuestionario.

El cuestionario era entregado por enfermeras y matronas en la planta de puérperas del hospital; las madres lo auto cumplimentaban antes del alta hospitalaria $\left(2^{\circ}-5^{\circ}\right.$ día) y lo dejaban en el control en el momento del alta. Para minimizar la pérdida de datos en la recogida de encuestas si faltaba algún dato se completaba mediante la revisión de historias clínicas o en la llamada telefónica que se realizaba a los 2 meses para preguntarles el tipo de Lactancia en ese momento.

A pesar del esfuerzo por completar todos los datos del cuestionario quedan sin rellenar el inicio de lactancia en las 2 primeras horas y continuación a los 2 meses en 2 y 1 cuestionario respectivamente; a los 2 meses postparto se contactó telefónicamente con las madres para preguntarles el tipo de Lactancia en ese momento, realizando 5 llamadas en días consecutivos.

\section{Estrategia del análisis estadístico}

Se evaluó la asociación entre las variables con el test Chi cuadrado de Pearson mediante el cálculo del RR y su IC para cuantificar la magnitud del efecto. En todos los casos, se fijó un nivel de significación de p<0,05.

La variable cuantitativa edad se describió mediante la media y su desviación típica (DT), comprobándose la normalidad de su distribución mediante el test de Kolmogorov- Smirnof.

El análisis fue realizado con el software estadístico SPSS versión 15.0.

\section{Consideraciones éticas}

El Comité de ética e investigación Clínica del Hospital Miguel Servet de Zaragoza autorizó este trabajo, no 
identificando conflicto alguno para su realización; la revisión de las historias clínicas se realizó tras aprobarse la solicitud de revisión de información con fines docentes y de investigación; se asegura la confidencialidad, según las normas vigentes actualmente y de acuerdo con la Asociación Médica Mundial y la Declaración de Helsinki.

\section{Resultados}

Durante el periodo a estudio hubo 737 partos (16,30 \% del total del año), el 28,50 \% de los partos (210 partos) fueron de madre extranjera; no se entregó el cuestionario a 71 madres por cumplir criterios de exclusión. De los 666 cuestionarios entregados se recogieron 593, perdiéndose $73(10,96 \%)$.

A los 2 meses se intentó localizar telefónicamente a las 593 madres, 48 no fueron localizadas y 4 decidieron abandonar el estudio en ese momento. Por tanto, el estudio se realiza finalmente sobre 541 madres.

Se perdieron un total de 120 madres extranjeras ( $42,86 \%$ ) frente a 76 españolas $(14,42 \%)$, la mayoría de pérdidas en extranjeras se produjeron al no entregar el cuestionario en el control en el momento del alta y al no ser localizadas telefónicamente a los 2 meses (Figura 1).

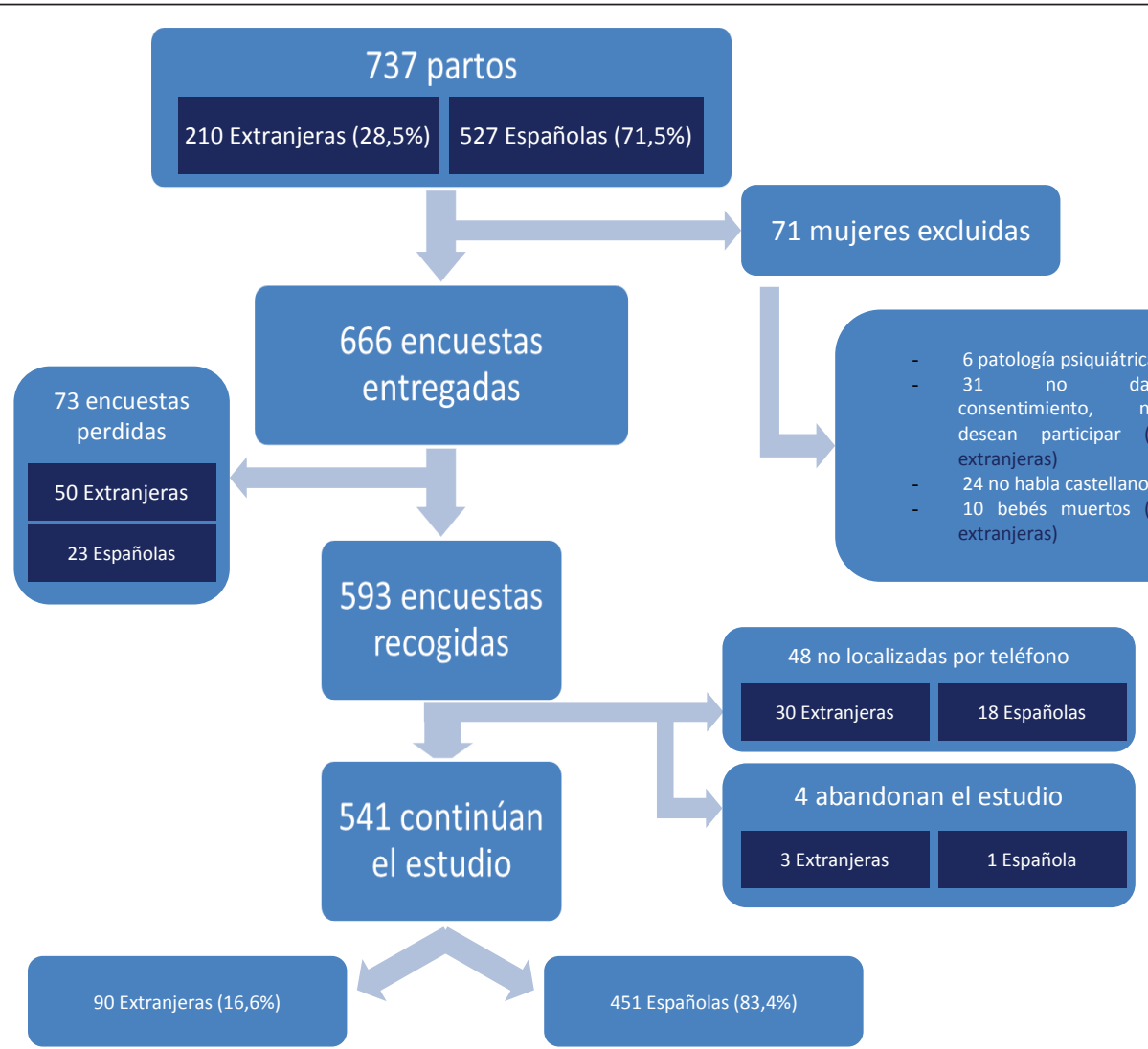

Figura 1. Diagrama de la toma de datos 
La población de estudio estaba formada por 451 madres españolas y 90 extranjeras (16,63\%), la media de edad fue 32,87 años, un 70,32\% de madres iniciaron lactancia en las dos primeras horas tras el parto y el 55,58 \% continuaron con LME a los dos meses (Tabla 1).

Tabla 1. Características generales de la muestra

\begin{tabular}{|c|c|c|}
\hline Edad & & $32,87 \pm 4,47^{*}$ \\
\hline Nacionalidad & $\begin{array}{l}\text { Española } \\
\text { Extranjeras }\end{array}$ & $\begin{array}{l}451 / 541(83,36 \%) \\
90 / 541(16,64 \%)\end{array}$ \\
\hline \multirow{5}{*}{ Tipo nacionalidad (\% sobre extranjeras) } & Latinoamericanas & $36 / 90(40 \%)$ \\
\hline & Europa Este & $28 / 90(31,11 \%)$ \\
\hline & Magrebí y subsahariana & $18 / 90(20 \%)$ \\
\hline & Chinas & $3 / 90(3,33 \%)$ \\
\hline & Europa UE (Italia, Francia, Inglaterra) & $5 / 90(5,56 \%)$ \\
\hline \multirow{4}{*}{ Tipo de parto } & Parto normal & $376 / 541(69,50 \%)$ \\
\hline & Parto Distócico & $165 / 541(30,50 \%)$ \\
\hline & Parto instrumental & $84 / 541(14,97 \%)$ \\
\hline & Cesárea & $81 / 541(15,53 \%)$ \\
\hline Contacto precoz en la primera hora & & $395 / 541(73,01 \%)$ \\
\hline Prematuridad & & $59 / 541(10,91 \%)$ \\
\hline Lactancia materna en las dos primeras horas & & $379 / 539(70,32 \%)$ \\
\hline \multirow{2}{*}{ Tipo de lactancia a los 2 meses postparto } & Lactancia materna exclusiva & $301 / 540(55,74 \%)$ \\
\hline & Lactancia mixta y /o artificial & $239 / 540(44,26 \%)$ \\
\hline
\end{tabular}

* Media \pm desviación típica

Se analizaron los posibles factores explicativos de iniciación de lactancia en las 2 primeras horas tras el parto y los de continuación con LME a los 2 meses postparto (Tabla 2a y Tabla 2b); se asociaron con el inicio precoz de lactancia materna las variables tipo de parto (RR 1,74; IC 95\% 1,46-2,07; p < 0,001), contacto precoz piel con piel madre- recién nacido (RR 3,65; IC 95\% $2,72-4,88 ; \mathrm{p}<0,001$ ) y parto prematuro (RR 2,71 ; IC $95 \%$ 2,14-3,42; $<<0,001$ ), se relacionaron con continuación de LME a los dos meses postparto todas las variables estudiadas: Nacionalidad (RR 1,28; IC $95 \%$ 1,01-1,64; $\mathrm{p}=0,02$ ), tipo de parto (RR 1,25; IC 95\% 1,04-1,50; $\mathrm{p}<0,001$ ), contacto precoz madre-recién nacido ( $R R$ 1,27 ; IC $95 \% 1,04-1,53 ; \mathrm{p}<0,001$ ) y parto prematuro (RR 1,54; IC 1,24-1,91; $<<0,001$ ); al referirse a las variables inicio de lactancia en las 2 horas postparto y el LME a los 2 meses, se comprobó la existencia de diferencias estadísticamente significativas (RR 1,72; IC 95\% 1,382,14; P < 0.001) (Tabla 3). 
Tabla 2a. Relación lactancia materna y factores asociados

\begin{tabular}{|c|c|c|c|c|c|c|}
\hline \multirow[t]{2}{*}{ Variables predictoras } & \multicolumn{4}{|c|}{$\begin{array}{l}\text { Lactancia materna } 2 \text { primeras horas } \\
\text { postparto }\end{array}$} & \multirow{2}{*}{$\begin{array}{l}\text { Significación } \\
\text { estadística (p) }\end{array}$} & \multirow{2}{*}{$\begin{array}{l}\text { Riesgo relativo } \\
\text { (IC } 95 \%)\end{array}$} \\
\hline & \multicolumn{2}{|c|}{ Sí } & \multicolumn{2}{|c|}{ No } & & \\
\hline \multicolumn{5}{|l|}{ Nacionalidad } & \multirow{3}{*}{0,58} & \\
\hline Española & 315 & $69,80 \%$ & 136 & $30,20 \%$ & & \\
\hline Extranjera & 64 & $72,70 \%$ & 24 & $27,30 \%$ & & \\
\hline \multicolumn{5}{|l|}{ Tipo de parto } & \multirow{3}{*}{$<0,001^{*}$} & \multirow{3}{*}{$\begin{array}{c}1,74 \\
(1,46-2,07)\end{array}$} \\
\hline Eutócico & 303 & $80,80 \%$ & 72 & $19,20 \%$ & & \\
\hline Distócico & 76 & $46,30 \%$ & 88 & $53,70 \%$ & & \\
\hline \multicolumn{5}{|l|}{ Contacto piel con piel } & \multirow{3}{*}{$<0,001^{*}$} & \multirow{3}{*}{$\begin{array}{c}3,65 \\
(2,72-4,88)\end{array}$} \\
\hline $\mathrm{Si}$ & 344 & $87,50 \%$ & 49 & $12,50 \%$ & & \\
\hline No & 35 & $24,00 \%$ & 111 & $76,00 \%$ & & \\
\hline \multicolumn{5}{|l|}{ Parto prematuro } & \multirow{3}{*}{$<0,001^{*}$} & \multirow{3}{*}{$\begin{array}{c}2,17 \\
(12,14-3,42)\end{array}$} \\
\hline Sí & 19 & $32,20 \%$ & 40 & $67,80 \%$ & & \\
\hline No & 360 & $75,00 \%$ & 120 & $25,00 \%$ & & \\
\hline
\end{tabular}

Tabla 2b. Relación lactancia materna y factores asociados

\begin{tabular}{|c|c|c|c|c|c|c|}
\hline \multirow{2}{*}{ Variables predictoras } & \multicolumn{4}{|c|}{$\begin{array}{c}\text { Lactancia materna exclusiva en los } 2 \\
\text { meses postparto }\end{array}$} & \multirow{2}{*}{$\begin{array}{l}\text { Significación } \\
\text { estadística (p) }\end{array}$} & \multirow{2}{*}{$\frac{\text { Riesgo relativo }}{\text { (IC } 95 \%)}$} \\
\hline & & Sí & & No & & \\
\hline \multicolumn{5}{|l|}{ Nacionalidad } & \multirow{3}{*}{$0,02^{*}$} & \multirow{3}{*}{$\begin{array}{c}1,28 \\
(1,01-1,64)\end{array}$} \\
\hline Española & 261 & $57,90 \%$ & 190 & $42,10 \%$ & & \\
\hline Extranjera & 40 & $44,90 \%$ & 49 & $55,10 \%$ & & \\
\hline \multicolumn{5}{|l|}{ Tipo de parto } & \multirow{3}{*}{$<0,001^{*}$} & \multirow{3}{*}{$\begin{array}{c}1,25 \\
(1,04-1,50)\end{array}$} \\
\hline Eutócico & 223 & $59,50 \%$ & 152 & $40,50 \%$ & & \\
\hline Distócico & 78 & $47,30 \%$ & 87 & $52,70 \%$ & & \\
\hline \multicolumn{5}{|l|}{ Contacto piel con piel } & \multirow{3}{*}{$<0,001^{*}$} & \multirow{3}{*}{$\begin{array}{c}1,27 \\
(1,04-1,53)\end{array}$} \\
\hline $\mathrm{Si}$ & 233 & $59,10 \%$ & 161 & $40,90 \%$ & & \\
\hline No & 68 & $46,60 \%$ & 78 & $53,40 \%$ & & \\
\hline \multicolumn{5}{|l|}{ Parto prematuro } & \multirow{3}{*}{$<0,001^{*}$} & \multirow{3}{*}{$\begin{array}{c}1,54 \\
(1,24-1,91)\end{array}$} \\
\hline Sí & 21 & $35,60 \%$ & 38 & $64,40 \%$ & & \\
\hline No & 280 & $58,20 \%$ & 201 & $41,80 \%$ & & \\
\hline
\end{tabular}

* Diferencias estadísticamente significativas 
Tabla 3. Relación lactancia materna en las 2 primeras horas y lactancia materna exclusiva a los 2 meses

\begin{tabular}{|c|c|c|c|c|c|c|}
\hline \multirow[t]{2}{*}{ Variables predictoras } & \multicolumn{4}{|c|}{$\begin{array}{c}\text { Lactancia materna exclusiva a los } 2 \\
\text { meses postparto }\end{array}$} & \multirow{2}{*}{$\begin{array}{l}\text { Significación } \\
\text { estadística (p) }\end{array}$} & \multirow{2}{*}{$\begin{array}{l}\text { Riesgo relativo } \\
\text { (IC } 95 \% \text { ) }\end{array}$} \\
\hline & \multicolumn{2}{|c|}{ Sí } & \multicolumn{2}{|c|}{ No } & & \\
\hline \multicolumn{5}{|l|}{ Lactancia materna precoz } & \multirow{3}{*}{$<0,001^{*}$} & \multirow{3}{*}{$\begin{array}{c}1,72 \\
(1,39-2,14)\end{array}$} \\
\hline SI & 240 & $63,50 \%$ & 138 & $36,50 \%$ & & \\
\hline No & 59 & $36,90 \%$ & 101 & $63,10 \%$ & & \\
\hline
\end{tabular}

Respecto a las distintas nacionalidades no existían diferencias estadísticamente significativas con inicio de lactancia, pero si con continuidad de la LME a los 2 meses, existiendo asociación estadísticamente significativa con las nacionalidades de Europa del Este (RR 2,32; IC 95\% 1,553,$45 ; \mathrm{p}<0,001$ ) y Latinoamérica (RR 1,56; IC 96\% 1,06-2,30; $\mathrm{p}=0,02$ ) (Tabla 4a y Tabla 4b).

Tabla 4a. Relación lactancia materna y tipo de nacionalidad

\begin{tabular}{|c|c|c|c|c|c|c|}
\hline \multirow{2}{*}{ Variables predictoras } & \multicolumn{4}{|c|}{ Lactancia materna en las 2 primeras horas postparto } & \multirow{2}{*}{$\begin{array}{l}\text { Significación } \\
\text { estadística (p) }\end{array}$} & \multirow{2}{*}{$\begin{array}{c}\text { Riesgo relativc } \\
\text { (IC 95\%) } \\
\end{array}$} \\
\hline & \multicolumn{2}{|c|}{ Sí } & \multicolumn{2}{|r|}{ No } & & \\
\hline \multicolumn{5}{|l|}{ Europa del Este } & \multirow{3}{*}{0,28} & \\
\hline SI & 23 & $82,10 \%$ & 5 & $17,90 \%$ & & \\
\hline NO & 40 & $71,40 \%$ & 16 & $28,60 \%$ & & \\
\hline \multicolumn{5}{|l|}{ Latinoamericanas } & \multirow{3}{*}{0,29} & \\
\hline SI & 26 & $81,30 \%$ & 6 & $18,80 \%$ & & \\
\hline NO & 37 & $71,20 \%$ & 15 & $28,80 \%$ & & \\
\hline \multicolumn{5}{|l|}{ Magrebíes y subsaharianas } & \multirow{3}{*}{0,52} & \\
\hline SI & 11 & $68,80 \%$ & 5 & $31,30 \%$ & & \\
\hline NO & 52 & $76,50 \%$ & 16 & $23,50 \%$ & & \\
\hline
\end{tabular}

Tabla 4b. Relación lactancia materna y tipo de nacionalidad

\begin{tabular}{|c|c|c|c|c|c|c|}
\hline \multirow{2}{*}{ Variables predictoras } & \multicolumn{4}{|c|}{ Lactancia materna a los 2 meses postparto } & \multirow{2}{*}{$\begin{array}{l}\text { Significación } \\
\text { estadística (p) }\end{array}$} & \multirow{2}{*}{$\begin{array}{c}\text { Riesgo relativo } \\
\text { (IC 95\%) }\end{array}$} \\
\hline & \multicolumn{2}{|c|}{ Sí } & \multicolumn{2}{|c|}{ No } & & \\
\hline \multicolumn{5}{|l|}{ Europa del Este } & \multirow{3}{*}{$0,001^{*}$} & \multirow{3}{*}{$\begin{array}{c}2,32 \\
(1,55-3,45)\end{array}$} \\
\hline SI & 22 & $78,60 \%$ & 6 & $21,40 \%$ & & \\
\hline NO & 21 & $33,90 \%$ & 41 & $66,10 \%$ & & \\
\hline \multicolumn{5}{|l|}{ Latinoamericanas } & \multirow{3}{*}{$0,02^{*}$} & \multirow{3}{*}{$\begin{array}{c}1,56 \\
(1,06-2,30)\end{array}$} \\
\hline SI & 12 & $33,30 \%$ & 24 & $66,70 \%$ & & \\
\hline NO & 31 & $57,40 \%$ & 23 & $42,60 \%$ & & \\
\hline \multicolumn{5}{|l|}{ Magrebíes y subsaharianas } & \multirow{3}{*}{0,39} & \\
\hline SI & 7 & $38,90 \%$ & 11 & $61,10 \%$ & & \\
\hline NO & 36 & $50,00 \%$ & 36 & $50,00 \%$ & & \\
\hline
\end{tabular}

* diferencias estadísticamente significativas 


\section{Discusión}

En el año 2011 hubo en nuestro hospital un total de $14,90 \%$ cesáreas, $16,43 \%$ partos instrumentales y 9,12 $\%$ prematuros, según los datos de 2011 del Ministerio de Trabajo e Inmigración ${ }^{14}$ en las mujeres la nacionalidad más frecuente es la latinoamericana, seguidas de países de la Unión Europea (alrededor del 50 \%), después Rumanía (48,2\%) y Marruecos (40,8\%); en esta muestra la nacionalidad latinoamericana también es la más frecuente.

La principal dificultad para comparar los datos de este estudio con otros es debido a la diferente medición en los tiempos y criterios seguidos en la lactancia y la no especificación en algunos estudios de si la LM incluye también la lactancia mixta.

En este estudio sólo se han excluido a los abortos (menos de 22 semanas edad gestacional) por tanto existe una dificultad en relación a la lactancia materna en los partos prematuros, siendo aquellos por debajo de la 37 semanas de edad gestacional, clasificándose según Lumley $^{17}$ en: prematuridad extrema (20-27 semanas), moderada (28-31 semanas) o leve (32-36 semanas). A los prematuros sin reflejo de succión-deglución (este reflejo aparece alrededor de las 34 semanas de gestación) se les administra leche, preferiblemente materna, por SNG aunque según la experiencia de Gómez Papí ${ }^{18}$ a partir de la 31 semana puede realizar tomas al pecho, pero lógicamente no en las primeras 2 horas tras el parto.

En este estudio las 19 madres que iniciaron lactancia materna en las primeras 2 horas han sido de prematuros de más de 34 semanas edad gestacional, cuyas madres han podido realizar el método canguro con toma al pecho y extracción de calostro con sacaleches en caso de que el bebé estuviese en neonatos.

No se han querido excluir los prematuros de menos 34 semanas debido a que se consideran $\operatorname{partos}^{17} \mathrm{y}$ queríamos obtener mayor muestra de madres de otras nacionalidades diferentes a la española, además se desea que este artículo además de estudiar la repercusión de la nacionalidad, tipo de parto o contacto precoz en la lactancia materna sirva también de reflexión sobre el método canguro y la importancia de dar leche materna a los prematuros.

Dos estudios realizados en 2007 encontraron un porcentaje similar al de este estudio en el inicio de
LM, refiriendo $74,7 \%{ }^{19}$,y $76,7 \%{ }^{20}$ de LME, pero al alta hospitalaria; a los 2 meses porcentajes parecidos al nuestro son un $45,26 \%{ }^{21}$ y un estudio más reciente un $59,4 \%{ }^{22}$, ambos porcentajes son de LME al segundo mes.

El porcentaje de LME a los 2 meses disminuye un $14,74 \%$ respecto al inicio, en otros estudios disminuye hasta un $20,4 \%{ }^{6} \mathrm{e}$ incluso $23,05 \%{ }^{21}$, la causa de este menor descenso puede ser por la pérdida de madres al entregar las encuestas y contestarnos por teléfono, pudiendo darse la circunstancia de que lo hiciesen las más motivadas en lactancia, otro motivo puede ser la alta proporción de madres que realizan contacto precoz en nuestro hospital $(73,01 \%)$, realizándose en otros estudios a tan sólo el 5,3\% de los recién nacidos ${ }^{23}$.

Los resultados de este estudio muestran que existe más riesgo de no iniciar la lactancia en las dos primeras horas tras el parto así como no continuarla con LME a los 2 meses en los partos distócicos, si no se ha realizado el contacto piel con piel en la primera hora y en los partos prematuros.

Estos datos coinciden con otros estudios recientes ${ }^{1,16,20,24,25}$, reflejando también que todos estos factores influyen negativamente en la LM; también se demuestra que el inicio de la lactancia en las dos primeras horas se asocia con su continuación, en cuanto al tipo de parto, algún estudio no encuentra relación con el inicio de LM ${ }^{26}$. Romero et al. ${ }^{11}$ encuentra asociación, pero esta desaparece a medida que pasan los meses. En el tiempo de estudio la nacionalidad no influye en el inicio de la lactancia materna pero si en su continuación, las madres españolas tienen más probabilidad de LME a los 2 meses. Estos resultados contrastan con los datos nacionales de $2006^{3}$ y con la bibliografía consultada, por ejemplo, el estudio CALINA llevado a cabo en Aragón ${ }^{25}$, hay que indicar un estudio realizado en un hospital Andaluz en 2008 en el que no se detectaron diferencias en la nacionalidad de la madre (española o latinoamericana) ${ }^{27}$.

Al realizar el estudio comparativo de las distintas nacionalidades en la continuación de la lactancia, las madres de Europa del Este son las que tienen más probabilidades de continuar con LME(el doble de probabilidades respecto al resto de extranjeras) y las latinoamericanas las que menos (en Colombia la lactancia materna exclusiva a los 6 meses es en este país es baja a pesar de haber aumentado considerablemente la lactancia en la primera hora ${ }^{28}$ ); en otro estudio realizado en Aragón ${ }^{25}$, a diferencia del nuestro 
encuentran mayor prevalencia de lactancia en madres africanas coincidiendo con un estudio Murciano ${ }^{16 .}$

Este estudio demuestra la importancia del contacto precoz para el inicio de LM en las dos primeras horas y para continuar con LME. Se comprueba que en los casos en que se realiza una serie de prácticas hospitalarias que actúan de modo barrera para el contacto precoz (estos casos son cesáreas y recién nacidos de bajo peso o prematuros) existen menos probabilidades de inicio y mantenimiento de lactancia materna.

Destacar que en un estudio realizado en Aragón, al estudiar la LM al alta en recién nacidos por cesárea, tienen el doble de probabilidad de LM aquellos en los que se estableció contacto precoz ${ }^{29}$. Según Ros Mar y Ros $\mathrm{Arnal}^{30}$, las madres subsaharianas son las que más mantienen las normas de alimentación de sus países de origen. Hay que tener en cuenta la importancia de respetar las diferencias culturales pues en lugares donde las tasas de mortalidad perinatal son elevadas puede ser frecuente la vinculación tardía con el $\mathrm{RN}^{31}$.

Las madres inmigrantes pueden presentar unas características peculiares en la alimentación de los bebés ${ }^{30}$ : En algunas culturas les introducen más precozmente los alimentos (incluyendo la leche de vaca) y aunque el niño puede mamar durante años puede ser casi inconcebible la LME.

Resaltar los esfuerzos que se están realizando en algunos países, por ejemplo Colombia, para fomentar la lactancia materna, implementando políticas y estrategias a favor de la promoción, protección y apoyo a la lactancia materna ${ }^{32}$ consiguiendo mejorar el inicio de la lactancia materna en la primera hora del recién nacido, al pasar de $48.9 \%$ a $57 \%$ de 2005 a 2010. No ocurrió lo mismo con la lactancia materna exclusiva a los 6 meses y su duración total.

Por todo ello, los resultados de este estudio podrían haber cambiado si se hubiese estudiado la LM mixta o se hubiese continuado el estudio midiendo la prevalencia de LM en meses posteriores.

En este estudio no se han analizado si las gestantes no autóctonas pueden presentar más patología gestacional que conllevará mayores tasas de prematuridad y cesáreas, factores que también contribuirían a explicar el resultado encontrado; sería conveniente tenerlo en cuenta en posteriores estudios. También en futuras investigaciones se deberían analizar los motivos que influyen en el abandono de la LM en las diferentes nacionalidades, teniendo en cuenta que el comportamiento en la lactancia que presentan los inmigrantes en España dista mucho del que tendrían en su país de origen ${ }^{33}$ por el proceso de "aculturación" incorporando comportamientos similares a las españolas.

La limitación principal de nuestro estudio es la barrera lingüística, no contando con un intérprete, aunque el mayor número de pérdidas en inmigrantes ha sido durante el proceso de recogida de datos pudiendo deberse a una falta de apoyo socio-familiar.

En conclusión, este estudio tiene como implicación práctica intentar un cambio de rutinas hospitalarias principalmente en las cesáreas que permitan realizar contacto precoz e inicio de lactancia materna en las dos primeras horas, además de sensibilizarnos con los aspectos culturales en los partos que atendamos.

\section{Agradecimientos}

A todas las madres que han participado en este estudio y al personal de la planta de maternidad por su colaboración en la entrega de encuestas.

Agradecer el apoyo de la dirección de enfermería del HUMS y al Dr. Lapresta por su asesoramiento en el estudio.

\section{Referencias Bibliográficas}

1. Comité de lactancia materna de la AEP. Lactancia materna: guía para profesionales. Monografías de la AEP nº 5. Madrid: Ergón; 2004.

2. World Health Organization. Global Strategy for infant and Young Child Feeding.Ginebra. WHO, 2003.

3. Ministerio de Sanidad y Consumo. Encuesta Nacional de Salud de España 2006. Estilos de vida. Madrid: Ministerio de Sanidad y Consumo, 2007 [Consultado: 17 septiembre 2013. Disponible en: http://www.msc. es/estadEstudios/estadisticas/encuestaNacional/ encuestaIndice2006.htm.

4. Black RE, Allen LH, Bhutta ZA, Caulfield LE, De Onis M, Ezzati M. Maternal and child undernutrition study Group. Maternal and child undernutrition: global and regional exposures and health consequences. Lancet. 2008 Jan 19; 371(9608): 243-60.

5. Ras VE, Briones CO, Domingo FM. ¿Qué decide la madre, pecho o biberón?. Aten Primaria. 2005; 36 (5): 286. 
6. Oliver A, Torras N, Martínez L. Factores de influencia en la duración de la lactancia materna en el Hospital de Sant Pau de Barcelona. EnfermClin.. 2003; 6 (13): 329-36.

7. Hector D, King L, Webb K, HeYwood. Factors affecting breastfeeding practices: applying a conceptual framework. N S W public Health Bull. 2005 Mar-Apr; 16(3-4): 52-5.

8. OMS-UNICEF. Declaración de Innocenti (resolución WHA 45.34).WHO: Florencia, 1990 'citado el 10 de diciembre de 2012]. Disponible en: http://www.who. int/nutritionpublications/gs_infant_feeding_text_spa. pdf.

9. IHAN. [Consultado 8 de diciembre de 3013]. Disponible en: http:// www.ihan.es/index16.asp.

10. Aguayo MJ. Prácticas rutinarias en las salas de maternidad que favorecen la lactancia materna. En: Aguado Maldonado J. La lactancia materna. Sevilla: Universidad de Sevilla; 2001.

11. Romero ME, Algaba S, Albar MJ, Núñez E, Calero C, Pérez IM. Influencia de las prácticas hospitalarias en el inicio y el mantenimiento de la lactancia materna. Enfermclin. 2004; 14(4): 194-202.

12. Yngve A, Sjöströn M. Breastfeeding determinants and a suggested framework for action in Europe). PublicHealthNutr. 2001; 4 (2B): 729-39.

13. Instituto Nacional de Estadística. Indicadores demográficos básicos. Disponible en: http:// www. ine.es.

14. Extranjeros residentes en España a 30 de junio de 2011. Principales resultados. Madrid: Observatorio Permanente de la Inmigración. Ministerio de trabajo e Inmigración. 2001. Disponible en: http://extranjeros. empleo.gob.es/es/Estadisticas/operaciones/ con-certificado/201306/Residentes_Principales_ Resultados_30062013.pdf.

15. Instituto Aragonés de Estadística .Datos básicos de Aragón. Zaragoza: Gobierno de Aragón; 2012. Disponible en: http://www.aragon.es/estaticos/GobiernoAragon/ Organismos/Instituto AragonesEstadistica/ Documentos/docs/Areas/DatosBasic/2012/DBA2012_ Wb.pdf.

16. García MA, RosBO. Factores socioculturales y perinatales relacionados con la lactancia materna exclusiva. Enferm Clin.2010; 20(2):109-113.

17. Aguilar MT, Bajo J, Manzanares S. Parto pretérmino y prematuridad. Tratamiento de la amenaza y parto pretérmino. En: Arenas JM, Melchor Marcos JC, Mercé LT. Fundamentos de Obstetricia. $1^{\text {a }}$ ed. Madrid: SEGO; 2007.
18. Gómez PA. Lactancia materna en prematuros. Bol Pediatr 1997; 37: 147-152.

19. Peñuelas MC, Enjuto GB, Cruz AJ, Peñuelas CR. Prevalencia de la lactancia materna en atención primaria e influencia de factores sociosanitarios y culturales. Metas de enfermería. 2008; 11(7):14-17.

20. Gomis CR, Arellano MC, Parra HP, Calle JE, Oliver RA, GarcíaGR, Et al. Lactancia materna en la región de Murcia. ¿Seguimos con el problema? Acta Pediatr Esp. 2009; 67 (6):283-289.

21. Borja MC, Rico ME, Morales MM, Úbeda MI, Codoñer P. Estudio comparativo de la lactancia materna entre dos zonas básicas de salud de la Comunidad Valenciana. RevPediatr Aten Primaria. 2006; 8:421-33

22. Palomares MJ, Fabregat FE, Folch MS, Escrig GB, Escoín PF, Gil SC. Apoyo a la lactancia materna en una zona básica de salud; prevalencia y factores sociosanitarios relacionados. Revpediatr Aten primaria. 2012; XV [Consultado: diciembre 2013]; Disponible en http://www.pap.es/

23. Romero ME, Algaba PS, Albar MJ, Núñez OE, Calero BC, Pérez IM. Prevalencia de la lactancia materna. Metas enfermería. Octubre 2003; 59: 18-23

24. Barriuso LM. Estudio multivariante sobre la prevalencia de la lactancia materna en el centro-norte de España. RevPediatr Aten Primaria. 2007; 9:589-612.

25. Cuadrón AL, Samper MP, Álvarez ML, Lasarte JJ, Rodríguez MG. Prevalencia de la lactancia materna durante el primer año de vida en Aragón. Estudio CALINA. Anales de Pediatría. Noviembre 2013; 79(5): 312-318

26. Iglesias CS. Lactancia materna en un hospital comarcal: factores socioculturales y sanitarios que pueden influir en su elección y mantenimiento. EnfermClin. 2008; $8(3): 142-6$

27. Medina LR, Doncel MD, Reyes AS, Álvarez AJ, Morales JM. Prevalencia de la lactancia materna y factores asociados a la adherencia prolongada. Metas de Enferm. 2011; 4(8): 16-22.

28. Manrique M, Corredor Martínez N, Bernardo N. Por una niñez bien nutrida. Unicef 2004

29. Otal LS, Morera LL, Bernal MJ, Tabueña AJ. El contacto precoz y su importancia en la lactancia materna frente a la cesárea. Matronas Prof. 2012; 13(1):3-8.

30. Ros ML, Ros AI. Alimentación de los niños inmigrantres en España durante su primer año de vida [consultado el 12 diciembre 2012]. Disponible en: htpp:// www. nutricia-pediatra.com

31. Scheper HN. La muerte sin llanto. Violencia y vida cotidiana en Brasil. Barcelona, Ariel, 1997 
32. Organización Panamericana de la Salud, Ministerio de Salud y Protección Social (Colombia). Avances y retos de la lactancia materna: Colombia 1970 a 2013. Un recorrido sobre 40 años de experiencias en la aplicación de políticas públicas.2013
33. Rovillé SF, Prado C, Madorrán MC. Alimentación en niños de origen chino y marroquí nacidos en Francia. Nutr. Clin Diet Hosp. 2010; 30:44-51.

Para citar este artículo:

Laviña A. Influencia del contacto precoz, nacionalidad, tipo de parto y prematuridad en la lactancia materna. Duazary. 2014 Dic; 11 (2): 115 - 125 